\title{
LETTER
}

Przemysław Kościk • Radosław Maj

\section{Note on the Harmonic Approximation in the Treatment of Entanglement: $N$ Cold Trapped Ions}

Received: 26 September 2014 / Accepted: 8 October 2014 / Published online: 17 October 2014

(C) The Author(s) 2014. This article is published with open access at Springerlink.com

\begin{abstract}
We study a one-dimensional system composed of $N$ charged bosons confined in an external harmonic potential. In the limit of a strong interaction between the particles, we apply the harmonic approximation and derive an integral representation for the purity of the one-particle reduced density matrix, enabling an easy determination of the asymptotic entanglement. Results for the dependence of the asymptotic linear entropy on $N$ are provided and discussed in detail for the first time.
\end{abstract}

\section{Introduction}

In the past few years there has been considerable interest in exploring the properties of quantum systems of interacting particles in a confining potential. In particular, systems of repelling particles Coulombically held together in a harmonic trap have attracted much attention because of their usefulness in modeling various experimentally fabricated systems. In this respect, the best known are the quantum dots [1] or ions in electromagnetic traps [2], enabling, among other things, the formation of strongly correlated few-body Wigner crystal states [3]. Recently, a number of papers have also dealt with the entanglement properties of such systems [4-9], but, mainly, in the two-particle case.

Here we consider systems consisting of $N$ charged particles in a trap modeled by a one-dimensional harmonic potential

$$
H=-\frac{1}{2} \sum_{i=1}^{N} \frac{\partial^{2}}{\partial x_{i}^{2}}+U
$$

where

$$
U=\frac{1}{2} \sum_{i=1}^{N} x_{i}^{2}+\sum_{i>j=1}^{N} \frac{g}{\left|x_{i}-x_{j}\right|} .
$$

In a recent study [8], performed by one of us, the entanglement in the ground state of the three-particle system (1) $(N=3)$ was investigated in the $g \rightarrow \infty$ limit by applying the harmonic approximation [10,11]. In the present paper, we go one step further and, based on a method of [8], gain some insight into the effect of $N$ on the ground-state entanglement of (1) as $g \rightarrow \infty$.

This paper is organized as follows. In Sect. 2 we derive, within the framework of the harmonic approximation, an explicit integral representation for the purity of the asymptotic one-particle reduced density matrix $(g \rightarrow \infty)$. Section 3 is devoted to results regarding the dependence of the entanglement on $N$, and a brief summary of our conclusions is given in Sect. 4.

P. Kościk $(\bowtie) \cdot$ R. Maj

Institute of Physics, Jan Kochanowski University, ul. Świętokrzyska 15, 25-406 Kielce, Poland

E-mail: koscik@pu.kielce.pl 


\section{Method}

\subsection{The Harmonic Approximation}

Due to the long-range nature of the Coulomb interaction, the distances between the particles increase with increasing $g$. At large enough $g$, the particles localize themselves at equilibrium positions $\boldsymbol{r}_{\min }^{c}=$ $\left\{x_{1}^{c}, x_{2}^{c}, \ldots, x_{N}^{c}\right\}$, forming a string, and the potential (2) can be well approximated harmonically around this point [10], which gives the Hessian matrix

$$
\mathbf{H}=\left[\left.\frac{\partial^{2} U}{\partial x_{m} \partial x_{k}}\right|_{\left\{r=r_{m i n}^{c}\right\}}\right]_{N \times N},
$$

with

$$
\frac{\partial^{2} U}{\partial x_{m} \partial x_{k}}=\delta_{m k}+2 g \sum_{i>j=1}^{N} \frac{\left(\delta_{i m}-\delta_{j m}\right)\left(\delta_{i k}-\delta_{j k}\right)}{\left|x_{i}-x_{j}\right|^{3}},
$$

where $\boldsymbol{r}_{\min }^{c}$ are the solutions of the set of equations

$$
\frac{\partial U}{\partial x_{k}}=x_{k}-g \sum_{i>j=1}^{N} \frac{x_{i}-x_{j}}{\left|x_{i}-x_{j}\right|^{3}}\left(\delta_{i k}-\delta_{j k}\right)=0,
$$

$k=1, \ldots, N$. The classical equilibrium positions of the particles are proportional to $g^{1 / 3}$, that is, $x_{i}^{c}=\alpha_{i} g^{1 / 3}$, so that the Hessian (3) does not depend on $g$ but only on the set of parameters $\alpha_{i}[10]$. From here on we refer to the point $\boldsymbol{r}_{\text {min }}^{c}$ with $x_{1}^{c}<x_{2}^{c}<\ldots<x_{N}^{c}$, which in the cases of even and odd values of $N$ has the form $\boldsymbol{r}_{\text {min }}^{c}=g^{1 / 3}\left\{-\alpha_{1},-\alpha_{2}, \ldots,-\alpha_{(N / 2)-1}, \alpha_{N / 2}, \alpha_{(N / 2)-1}, \ldots, \alpha_{2}, \alpha_{1}\right\}$ and $\boldsymbol{r}_{\text {min }}^{c}=$ $g^{1 / 3}\left\{-\alpha_{1},-\alpha_{2}, \ldots,-\alpha_{(N-1) / 2}, 0, \alpha_{(N-1) / 2} \ldots, \alpha_{2}, \alpha_{1}\right\}\left(\alpha_{(N+1) / 2}=0\right)$, respectively [10] $\left(x_{i}^{c}=x_{N-i+1}^{c}\right)$. Within the standard normal mode theory $[10,11]$, the problem simplifies to a set of $N$ uncoupled oscillators in terms of the normal-mode coordinates $v_{i}, \omega_{i}^{2} v_{i}^{2} / 2$, which are related to $\boldsymbol{Z}=\left\{z_{1}, z_{2}, \ldots, z_{N}\right\}=\boldsymbol{r}-\boldsymbol{r}_{\min }^{c}$ by $\left\{v_{1}, v_{2}, \ldots, v_{N}\right\}=\mathbf{U} \boldsymbol{Z}$, where $\mathbf{U}$ is a unitary matrix that diagonalizes the Hessian $\mathbf{H}, \mathbf{H}^{\prime}=\mathbf{U} \mathbf{H U}^{T}$ and the normal mode frequencies $\omega_{i}$ are given by $\omega_{i}=\sqrt{H_{i i}^{\prime}}$.

An harmonic approximation to the bosonic ground state of (1) can be constructed as follows [8]:

$$
\Psi\left(x_{1}, x_{2}, \ldots, x_{N}\right)=C \sum_{s} \psi\left(x_{S(1)}-x_{1}^{c}, x_{s(2)}-x_{2}^{c}, \ldots, x_{S(N)}-x_{N}^{c}\right),
$$

with

$$
\psi\left(z_{1}, z_{2}, \ldots, z_{N}\right)=\prod_{i=1}^{N}\left(\frac{\sqrt{\omega_{i}}}{\pi}\right)^{\frac{1}{4}} e^{-\frac{\omega_{i} \nu_{i}^{2}\left(z_{1}, z_{2}, \ldots, z_{N}\right)}{2}},
$$

$\langle\psi \mid \psi\rangle=1$, where $\{s(1), s(2), \ldots, s(N)\}$ are the integers $\{1,2,3, \ldots, N\}$ permuted into a different order and $C$ is the normalization constant. It can be readily inferred at this point that in the asymptotic regime as $g \rightarrow \infty$, where $\gamma_{i j}=\left|x_{i}^{c}-x_{j}^{c}\right| \rightarrow \infty(i \neq j)$, the value of $C$ tends to $N !^{-1 / 2}, C^{g \rightarrow \infty}=N !^{-1 / 2}$.

\subsection{The Purity of the One-Particle Reduced Density Matrix}

Now we come to the point where we explore the effect of $N$ on the entanglement at the $g \rightarrow \infty$ limit. Most of the popular entanglement measures are functions of the purity $(\mathrm{P})$ of the one-particle reduced density matrix (1-RDM) $\hat{\rho}=\operatorname{Tr}_{2,3, . ., N}|\Psi\rangle\langle\Psi|$,

$$
\mathrm{P}=\operatorname{Tr} \hat{\rho}^{2}
$$


Among them, the best known are the linear entropy $\mathrm{L}=1-\mathrm{P}[12]$ and the participation ratio $\mathrm{R}=\mathrm{P}^{-1}[13]$. We recall here that the 1-RDM is used to characterize the bipartite entanglement between the subsets of one particle and the remaining $N-1$ particles [14]. The 1-RDM expressed in coordinates takes the form

$$
\rho(x, y)=\int_{-\infty}^{\infty} \ldots \int_{-\infty}^{\infty} \Psi\left(x, x_{2}, \ldots, x_{N}\right) \Psi\left(y, x_{2}, \ldots, x_{N}\right) d x_{2} d x_{3}, \ldots, d x_{N}
$$

and its purity can be calculated as [13]

$$
\mathrm{P}=\int_{-\infty}^{\infty} \int_{-\infty}^{\infty}[\rho(x, y)]^{2} d x d y
$$

In the already cited [8], the asymptotic behavior of the 1-RDM was discussed in the case of $N=3$. It is a quite straightforward task to derive an asymptotic form of the 1-RDM in the general case of $N$ particles. Indeed, after substituting (6) into (9), one easily infers that in the limit as $g \rightarrow \infty\left(\gamma_{i j} \rightarrow \infty\right)$, the 1-RDM reduces to

$$
\rho^{g \rightarrow \infty}=\rho_{1}+\rho_{2}+\ldots+\rho_{N}
$$

with

$$
\rho_{1}=D \int_{-\infty}^{\infty} \ldots \int_{-\infty}^{\infty} \psi\left(x-x_{1}^{c}, z_{2}, \ldots, z_{N}\right) \psi\left(y-x_{1}^{c}, z_{2}, \ldots, z_{N}\right) d z_{2} d z_{3}, \ldots, d z_{N}
$$

and

$$
\rho_{2}=D \int_{-\infty}^{\infty} \ldots \int_{-\infty}^{\infty} \psi\left(z_{1}, x-x_{2}^{c}, \ldots, z_{N}\right) \psi\left(z_{1}, y-x_{2}^{c}, \ldots, z_{N}\right) d z_{1} d z_{3} \ldots, d z_{N}
$$

$$
\rho_{N}=D \int_{-\infty}^{\infty} \ldots \int_{-\infty}^{\infty} \psi\left(z_{1}, z_{2}, \ldots, x-x_{N}^{c}\right) \psi\left(z_{1}, z_{2}, \ldots, y-x_{N}^{c}\right) d z_{1} d z_{2}, \ldots, d z_{N-1} .
$$

where $D=(N-1) !\left[C^{g \rightarrow \infty}\right]^{2}=N^{-1}$. Furthermore, an easy inspection of the normal-mode coordinates $v_{i}\left(z_{1}, \ldots, z_{N}\right)$ reveals that $\operatorname{Tr} \rho_{i}^{2}=\operatorname{Tr} \rho_{N-i+1}^{2}$ (for details concerning the properties of $v_{i}\left(z_{1}, \ldots, z_{N}\right)$, see, for example, [10]). Hence, bearing in mind this finding and the fact that the integral overlap between $\rho_{i}$ and $\rho_{j}$ vanishes for any $i \neq j$ as $g \rightarrow \infty$, we conclude that the asymptotic purity $\mathrm{P}^{g \rightarrow \infty}$ can be decomposed as

$$
\mathrm{P}^{g \rightarrow \infty}=2 \sum_{i=1}^{\frac{N}{2}} \operatorname{Tr} \rho_{i}^{2},(N-\text { even })
$$

and

$$
\mathrm{P}^{g \rightarrow \infty}=2 \sum_{i=1}^{\frac{N-1}{2}} \operatorname{Tr} \rho_{i}^{2}+\operatorname{Tr} \rho_{\frac{N+1}{2}}^{2},(N-\text { odd })
$$

with

$$
\operatorname{Tr} \rho_{i}^{2}=\int_{-\infty}^{\infty} \int_{-\infty}^{\infty} \tilde{\rho}_{i}^{2} d \tilde{x} d \tilde{y}
$$

where we have eliminated the equilibrium positions in $\rho_{i}$ by translating the coordinates by $x \mapsto \tilde{x}+x_{i}^{c}$, $y \mapsto \tilde{y}+x_{i}^{c},\left(\rho_{i} \mapsto \tilde{\rho}_{i}\right)$. 


\section{Results}

The classical equilibrium positions of the particles are known analytically only for $N=2,3$ [10]. For larger $N$, we numerically solve the set of (5) for the values of $x_{i}^{c}$. The integrals in (11) and in (14) can be performed in an algebraic way, but for the reasons mentioned above, the values of $\mathrm{P}^{g \rightarrow \infty}$ can be obtained in closed analytic form solely for $N=2,3$, that is,

$$
\mathrm{P}_{N=2}^{g \rightarrow \infty}=\sqrt{-\frac{3}{2}+\sqrt{3}} \approx 0.481
$$

and

$$
\mathrm{P}_{N=3}^{g \rightarrow \infty}=\frac{1}{3 \sqrt{5+\frac{68}{\sqrt{145}}}}+\frac{2}{3 \sqrt{\frac{7}{2}+2 \sqrt{3}+\sqrt{\frac{2}{145}(326+187 \sqrt{3})}}} \approx 0.313
$$

For illustrative purposes, we present in Table 1 some values of $\mathrm{L}^{g \rightarrow \infty}=1-\mathrm{P}^{g \rightarrow \infty}$ and of $\mathrm{R}^{g \rightarrow \infty}=\left(\mathrm{P}^{g \rightarrow \infty}\right)^{-1}$ obtained by us with the use of (12)-(13). In [9], the values of $L^{g \rightarrow \infty}$ were found numerically to be about $0.52,0.68$, and 0.77 for $N=2,3$ and $N=4$, respectively, where they were determined by the configuration interaction method. We find that the results obtained from (12)-(13) compare well with the above values, which confirms the correctness of our theoretical derivations.

We close with a brief discussion of the effect of $N$ on the entanglement. Figure 1 displays the dependence of $\mathrm{L}^{g \rightarrow \infty}$ for $N$ up to $N=10$, where in order to gain some insight into the effect of $g$, the variations of $\mathrm{L}$ for some exemplary values of $g$ are also depicted (these results are taken from Fig. 6 of [9]). The further extension of the results for $\mathrm{L}^{g \rightarrow \infty}$ for larger $N$ is a straightforward task but the computational time increases considerably. As may be seen from Fig. 1, the linear entropy L generally has a monotonically increasing behaviour as the number of particles in the system increases. In other words, as $N$ increases, the entanglement between the subsets of one particle and the remaining $N-1$ particles increases as well. As can be further inferred from Fig. 1, the larger is $N$, the smaller is the change in the entanglement (in a relative sense) produced by the addition of one particle to the system.

Finally, there may be a general interest in noting that the entanglement in the system (1) exhibits the opposite behaviour to that in a system of harmonically trapped bosons interacting via $V=g_{m}\left(x_{i}-x_{j}\right)^{2}$ (the

Table $1 L^{g \rightarrow \infty}$ and $R^{g \rightarrow \infty}$ determined as discussed in the text are compared with the numerical results of [9]

\begin{tabular}{llllll}
\hline & $N=2$ & $N=3$ & $N=4$ & $N=5$ & $N=6$ \\
\hline $\mathrm{L}^{g \rightarrow \infty}$ & 0.518283 & 0.686583 & 0.769126 & 0.817901 & 0.85001 \\
{$[9]$} & 0.52 & 0.68 & 0.77 & & \\
$\mathrm{R}^{g \rightarrow \infty}$ & 2.07591 & 3.19064 & 4.33137 & 5.49152 & 6.66711 \\
\hline
\end{tabular}

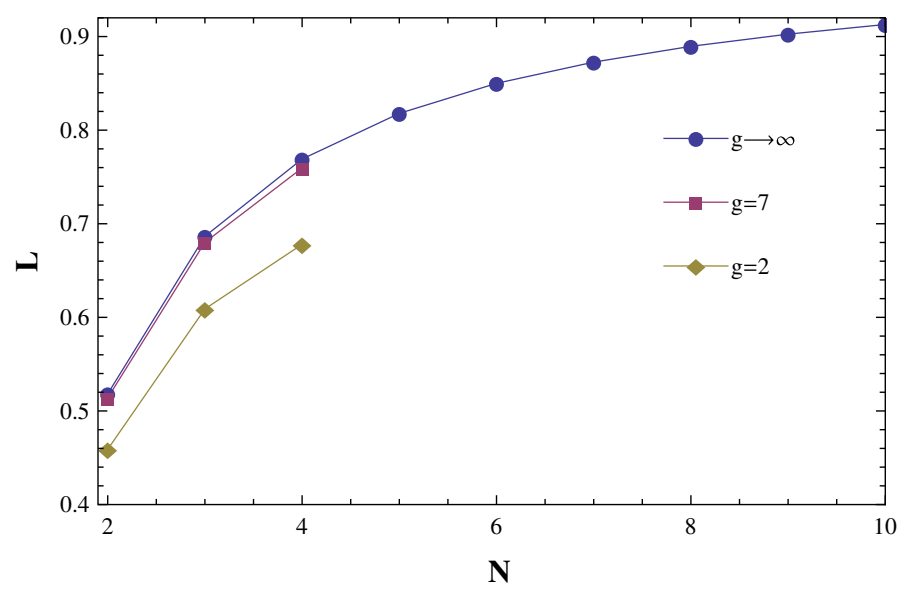

Fig. 1 The linear entropy L as a function of $N$ for $g=2,7, \infty$ 
so-called Moshinsky atom), where the ground-state linear entropy L decreases with $N$ for large enough $N$ [15]. The only exceptions here are the cases $g_{m}=0$ and $g_{m} \rightarrow \infty$ where $\mathrm{L}=0$ and $\mathrm{L}=1$, respectively, regardless of $N$.

\section{Summary}

We studied the entanglement properties of a system of $N$ charged bosons confined in a one-dimensional harmonic potential. We derived, within the framework of the harmonic approximation, an asymptotic integral expression for the purity of the one-particle reduced density matrix, allowing for its easy determination over a wide range of values of $N$. Based on this finding we revealed for the first time the effect of $N$ on the amount of entanglement in the asymptotic ground state of the system. Among other things, our results showed that the entanglement increases monotonically with $N$, making its most rapid variations in the regime of small values of $N$. It turned out that the main entanglement features exhibited by the present system are substantially different from those exhibited by the Moshinsky model system. It would be desirable to extend the current calculations to systems of higher spatial-dimensionality, and this is an open topic for future investigations.

Open Access This article is distributed under the terms of the Creative Commons Attribution License which permits any use, distribution, and reproduction in any medium, provided the original author(s) and the source are credited.

\section{References}

1. Jacak, L., Hawrylak, P., Wojs, A.: Quantum Dots. Springer-Verlag, Berlin (1977)

2. Wineland, D.J., et al.: Atomic-ion coulomb clusters in an ion trap. Phys. Rev. Lett. 59, 2935 (1987)

3. Filinov, A., Bonitz, M., Lozovik, Y.E.: Wigner crystallization in mesoscopic 2D electron systems. Phys. Rev. Lett. 86, 3851 (2001)

4. Coe, J.P., Sudbery, A., D'Amico, I.: Entanglement and density-functional theory: Testing approximations on Hooke's atom. Phys. Rev. B 77, 205122 (2008)

5. Nazmitdinov, R., et al.: Shape transitions in excited states of two-electron quantum dots in a magnetic field. J. Phys. B At. Mol. Opt. Phys. 45, 205503 (2012)

6. Nazmitdinov, R., et al.: A geometrical crossover in excited states of two-electron quantum dots in a magnetic field. J. Phys. Conf. Ser. 393, 012009 (2012)

7. Kościk, P.: Two-electron entanglement in a two-dimensional isotropic harmonic trap: Radial correlation effects in the low density limit. Phys. Lett. A 375, 458 (2011)

8. Kościk, P.: Three strongly correlated charged bosons in a one-dimensional harmonic trap: natural orbital occupancies. Eur. Phys. J. B 85, 173 (2012)

9. Kościk, P., Okopińska, A.: Ground-state correlation properties of charged bosons trapped in strongly anisotropic harmonic potentials. Eur. Phys. J. B 85, 93 (2012)

10. James, D.F.V.: Quantum dynamics of cold trapped ions with application to quantum computation. Appl. Phys. B 66, 181 (1998)

11. Balzer, K., et al.: Energy spectrum of strongly correlated particles in quantum dots. J. Phys. Conf. Ser. 35, 209 (2006)

12. Manfredi, G., Feix, M.: Entropy and Wigner functions. Phys. Rev. E 62, 4665 (2000)

13. Grobe, R., Rzazewski, K., Eberly, J.: Measure of electron-electron correlation in atomic physics. J. Phys. B At. Mol. Opt. Phys. 27, L503 (1994)

14. Borras, A., Majtey, A.P., Casas, M.: Efficient generation of random multipartite entangled states using time-optimal unitary operations. Phys. Rev. A 78, 022328 (2008)

15. Kościk, P., Okopińska, A.: Correlation effects in the Moshinsky model. Few-Body Syst. 54, 1637 (2013) 